\title{
Adjuvant therapies in critical care: steroids in community-acquired pneumonia
}

\author{
Antoni Torres ${ }^{1,2,3^{*}}$ (D), Miquel Ferrer ${ }^{1,2,3}$ and Michael S. Niederman ${ }^{4}$
}

๑ 2017 Springer-Verlag GmbH Germany and ESICM

Severe community-acquired pneumonia (CAP) is present in up to $19 \%$ of hospitalized patients with CAP and still carries a high morbidity and mortality. A French multicentre study of severe pneumococcal CAP patients admitted to intensive care units (ICU) reported an overall mortality rate of $29 \%$ [1]. The high mortality of severe CAP occurs despite the fact that the majority of patients receive an early and adequate antibiotic treatment. This is probably due, in part, to an imbalanced and disproportionate local and systemic inflammatory response that contributes to impairment of gas exchange, sepsis and end-organ dysfunction.

In humans, intravenous corticosteroids attenuate the local and systemic inflammatory response [2]. In a model of Pseudomonas aeruginosa pneumonia in mechanically ventilated piglets, we observed a lower lung bacterial burden and less severe histological pneumonia in animals treated with corticosteroids plus ciprofloxacin than those who did not get corticosteroids [3].

Since 1955, multiple randomised clinical trials (RCTs) have assessed the efficacy of adjuvant treatment with systemic corticosteroids in patients with CAP. Among these, treatment with hydrocortisone resulted in a striking decrease in mortality in patients with severe CAP [4].

Until now, ten meta-analyses about corticosteroids in CAP have been published, with assessment of mortality [5-14]. Four focused on severe CAP $[5,7,12,13]$ and four others [6,9-11] analysed severe CAP as a subgroup. In seven of these eight meta-analyses that evaluated patients with severe CAP, mortality was significantly decreased, with odds ratios ranging from 0.21 to 0.64 . The length of stay was significantly reduced in the nine meta-analyses

\footnotetext{
*Correspondence: atorres@ub.edu

${ }^{1}$ Department of Pneumology, Respiratory Institute, Hospital Clinic of Barcelona, University of Barcelona (UB), Villarroel 170, 08036 Barcelona, Spain

Full author information is available at the end of the article
}

that assessed this outcome (in one it was not searched for [6]). Time to clinical stability was reduced by about 1 day in four meta-analyses [8-10,14], while this reduction was not estimated quantitatively in another one [11], and this information was not assessed in the remaining five studies. In five out of seven studies that reported the data, hyperglycemia was significantly more common in the treatment arm. Gastrointestinal bleeding was not increased by the use of corticosteroids in the six metaanalyses in which it was specifically investigated. Table 1 summarizes the characteristics and findings of these meta-analyses.

Despite the benefits observed in the meta-analyses and a reasonable frequency of side effects, the use of corticosteroids in CAP is still controversial and not routine. There are pitfalls in some of the RCTs included in these meta-analyses. First, the inclusion of patients with low severity may not be informative, since these patients have a low mortality and consequently it is very difficult to demonstrate differences in important outcomes such as treatment failure and mortality. Second, while critical illness-related corticosteroid insufficiency was not systematically assessed in these studies [15], few limited inclusion to those with high initial degrees of systemic inflammation. According to the rationale of using steroids in CAP, a high inflammatory response is necessary to benefit from this treatment. Patients with a high inflammatory response, i.e. high levels of C-reactive protein (CRP), have higher rates of treatment failure and mortality [16]. Third, the dosages, the type and the length of treatment are very different among RCTs, which makes it very difficult to establish comparisons among them. Fourth, the primary endpoints are different between studies and some of them, such as length of stay or even time to clinical stability, could be considered "soft", since the former depends on other variables and the latter is driven by the persistence of fever, which in

\section{Springer}




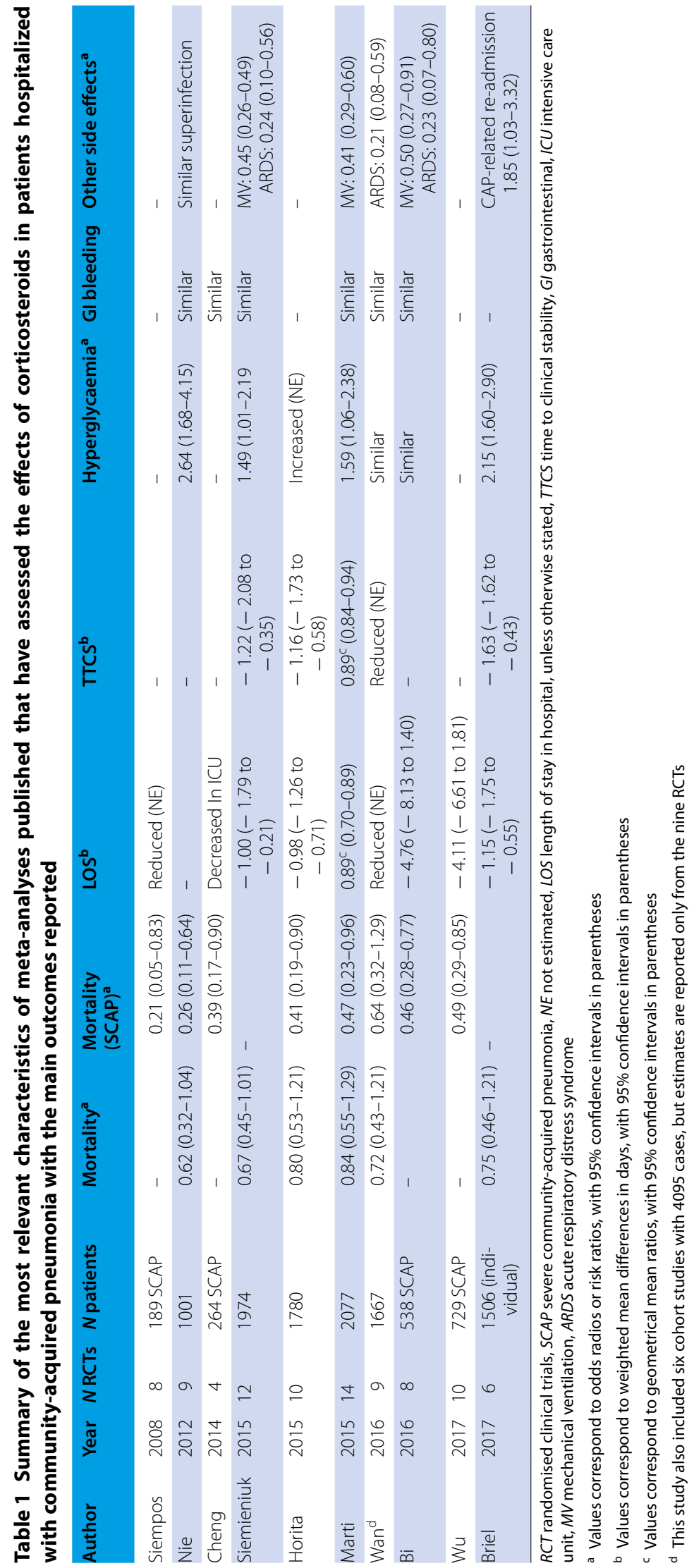


fact is downregulated by corticosteroids. Finally, it should be noted that the small sample size of some individual studies included in these meta-analyses is a limitation.

We performed a RCT [17] comparing methylprednisolone $(0.5 \mathrm{mg} / \mathrm{kg}$ b.i.d. during 5 days $)$ vs. placebo; but unlike other studies, we only included severe CAP patients and those with a high systemic inflammatory response, defined by CRP $>15 \mathrm{mg} / \mathrm{dL}$. Also, rather than using mortality as a primary endpoint, we chose treatment failure, since it is associated with higher mortality of CAP. We also monitored the systemic inflammatory response, using different biomarkers, until day 7 after the inclusion of patients in the trial.

Our results showed a decrease in the treatment failure rate. The reduction in treatment failure was more evident in late treatment failure, defined as radiographic progression or persistence of respiratory failure, development of shock, need for invasive mechanical ventilation not present at baseline or death between 72 and $120 \mathrm{~h}$ after treatment initiation, and was especially clear for radiographic progression. Mortality did not differ significantly between groups, but the study was not powered for this endpoint. Despite the fact that we did not observe a rebound inflammation 2 days after corticosteroid discontinuation in CAP, we cannot exclude such a complication after longer periods of treatment without tapering.

After reviewing all the meta-analyses published and considering our own findings, we believe that it is now time to start introducing treatment with corticosteroids to selected ICU patients with severe CAP. We recommend selecting severe CAP patients with a high inflammatory response, as measured by CRP, at similar dosage and duration as we used in our RCT [17]. Methylprednisolone, prednisone or hydrocortisone at equivalent dosage appear similarly effective. Despite the fact that we excluded patients with influenza pneumonia in our trial because of data suggesting that corticosteroids are associated with increased mortality in influenza pneumonia, this association may not be present when adequate adjustments are made for time-dependent differences, and therefore these patients probably should not be excluded from corticosteroid treatment. In the future, we may find that other biomarkers are more specific in identifying patients who most benefit from this therapy, and we may have new therapies that are more selective regarding their anti-inflammatory impact on pneumonia.

We also need more data on the possible effect of corticosteroids on other viral pneumonias caused by adenovirus, rhinovirus, respiratory syncytial virus or others such as MERS. On the basis of the available data, we believe that corticosteroids are useful in specific patients with severe CAP and can help to decrease length of stay, time to clinical stability, treatment failure and possibly mortality.

\section{Author details}

${ }^{1}$ Department of Pneumology, Respiratory Institute, Hospital Clinic of Barcelona, University of Barcelona (UB), Villarroel 170, 08036 Barcelona, Spain. ${ }^{2}$ Institut d'Investigacions Biomèdiques August Pi i Sunyer (IDIBAPS), Barcelona, Spain. ${ }^{3}$ Centro de Investigación Biomedica En Red-Enfermedades Respiratorias (CibeRes, CB06/06/0028), Barcelona, Spain. ${ }^{4}$ New York Presbyterian/Weill Cornell Medical Center, New York, NY, USA.

\section{Acknowledgements}

This study was supported by Grants SEPAR, FUCAP and SOCAP, 2009-SGR661, IDIBAPS, ICREA academia 2013, Instituto de Salud Carlos III (PI15/00506), CibeRes (CB06/06/0028)-ISCIII.

Received: 6 September 2017 Accepted: 11 October 2017

Published online: 25 October 2017

\section{References}

1. Mongardon N, Max A, Bougle A, Pene F, Lemiale V, Charpentier J, Cariou A Chiche JD, Bedos JP, Mira JP (2012) Epidemiology and outcome of severe pneumococcal pneumonia admitted to intensive care unit: a multicenter study. Crit Care 16:R155

2. Monton C, Ewig S, Torres A, El Ebiary M, Filella X, Rano A, Xaubet A (1999) Role of glucocorticoids on inflammatory response in nonimmunosuppressed patients with pneumonia: a pilot study. Eur Respir J 14:218-220

3. Sibila O, Luna CM, Agusti C, Baquero S, Gando S, Patron JR, Morato JG, Absi R, Bassi N, Torres A (2008) Effects of glucocorticoids in ventilated piglets with severe pneumonia. Eur Respir J 32:1037-1046

4. Confalonieri M, Urbino R, Potena A, Piattella M, Parigi P, Puccio G, Della PR, Giorgio C, Blasi F, Umberger R, Meduri GU (2005) Hydrocortisone infusion for severe community-acquired pneumonia: a preliminary randomized study. Am J Respir Crit Care Med 171:242-248

5. Siempos II, Vardakas KZ, Kopterides P, Falagas ME (2008) Adjunctive therapies for community-acquired pneumonia: a systematic review. J Antimicrob Chemother 62:661-668

6. Nie W, Zhang Y, Cheng J, Xiu Q (2012) Corticosteroids in the treatment of community-acquired pneumonia in adults: a meta-analysis. PLoS One 7:e47926

7. Cheng M, Pan ZY, Yang J, Gao YD (2014) Corticosteroid therapy for severe community-acquired pneumonia: a meta-analysis. Respir Care 59:557-563

8. Siemieniuk RA, Meade MO, Alonso-Coello P, Briel M, Evaniew N, Prasad M, Alexander PE, Fei Y, Vandvik PO, Loeb M, Guyatt GH (2015) Corticosteroid therapy for patients hospitalized with community-acquired pneumonia: a systematic review and meta-analysis. Ann Intern Med 163(7):519-528

9. Horita N, Otsuka T, Haranaga S, Namkoong H, Miki M, Miyashita N, Higa F, Takahashi H, Yoshida M, Kohno S, Kaneko T (2015) Adjunctive systemic corticosteroids for hospitalized community-acquired pneumonia: systematic review and meta-analysis 2015 update. Sci Rep 5:14061

10. Marti C, Grosgurin O, Harbarth S, Combescure C, Abbas M, Rutschmann O, Perrier A, Garin N (2015) Adjunctive corticotherapy for community acquired pneumonia: a systematic review and meta-analysis. PLoS One 10:e0144032

11. Wan YD, Sun TW, Liu ZQ, Zhang SG, Wang LX, Kan QC (2016) Efficacy and safety of corticosteroids for community-acquired pneumonia: a systematic review and meta-analysis. Chest 149:209-219

12. Bi J, Yang J, Wang Y, Yao C, Mei J, Liu Y, Cao J, Lu Y (2016) Efficacy and safety of adjunctive corticosteroids therapy for severe communityacquired pneumonia in adults: an updated systematic review and metaanalysis. PLoS One 11:e0165942

13. Wu WF, Fang Q, He GJ (2017) Efficacy of corticosteroid treatment for severe community-acquired pneumonia: a meta-analysis. Am J Emerg Med. doi:10.1016/.ajem.2017.07.050 
14. Briel M, Spoorenberg SMC, Snijders D, Torres A, Fernandez-Serrano S, Meduri GU, Gabarrús A, Blum CA, Confalonieri M, Kasenda B, Siemieniuk RAC, Boersma W, Bos WJW, Christ-Crain M, Ovidius study group, Capisce study group, STEP study group (2017) Corticosteroids in patients hospitalized with community-acquired pneumonia: systematic review and individual patient data meta-analysis. Clin Infect Dis. doi:10.1093/cid/ cix801

15. Annane D, Pastores SM, Rochwerg B, Arlt W, Balk RA, Beishuizen A, Briegel J, Carcillo J, Christ-Crain M, Cooper MS, Marik PE, Umberto MG, Olsen KM, Rodgers S, Russell JA, van den BG (2017) Guidelines for the diagnosis and management of critical illness-related corticosteroid insufficiency (CIRCI) in critically ill patients (Part I): Society of Critical Care Medicine (SCCM) and European Society of Intensive Care Medicine (ESICM) 2017. Intensive Care Med. doi:10.1097/CCM.0000000000002737
16. Menendez R, Martinez R, Reyes S, Mensa J, Filella X, Marcos MA, Martinez A, Esquinas C, Ramirez P, Torres A (2009) Biomarkers improve mortality prediction by prognostic scales in community-acquired pneumonia. Thorax 64:587-591

17. Torres A, Sibila O, Ferrer M, Polverino E, Menendez R, Mensa J, Gabarrus A, Sellares J, Restrepo MI, Anzueto A, Niederman MS, Agusti C (2015) Effect of corticosteroids on treatment failure among hospitalized patients with severe community-acquired pneumonia and high inflammatory response: a randomized clinical trial. JAMA 313:677-686 